\title{
Time-resolved measurements of the response of a STM tip upon illumination with a nanosecond laser pulse
}

\author{
J. Boneberg, M. Tresp, M. Ochmann, H.-J. Münzer, P. Leiderer
}

Fakultät für Physik, Universität Konstanz, D-78464 Konstanz, Germany (Fax: +49-7531/883091, E-mail: Johannes.Boneberg@uni-konstanz.de)

\begin{abstract}
Nanosecond laser pulses are used to illuminate the very end of a tip of a scanning tunneling microscope in front of a gold surface. The transient increase of the tunneling current is measured as a function of the pulse energy density, tip retraction amplitude, polarization of the incident light, and bias voltage. The transient signal has a typical timescale of ms. Thus it can be concluded that the thermal expansion of the tip is responsible for this signal. The expansion has a linear dependence on the incident light intensity with a typical value of the order of $0.1 \AA /\left(\mathrm{mJ} / \mathrm{cm}^{2}\right)$. This result demands a critical inspection of the interpretation of nanostructuring experiments with this technique.
\end{abstract}

PACS: $6116 \mathrm{P} ; 6570 ; 4260 \mathrm{~K}$

The scanning tunneling microscope and the atomic force microscope are frequently used for the production of nanostructures on surfaces. Whereas the possible use of forces or electric fields is already known from different applications [1] another quite new idea is the external injection of laser radiation into the tip-substrate gap. It has been shown that by using this technique nanostructures of different forms can be produced [2-6]. Even reliable single-atom deposition is achievable [5]. The physical reasons for these possibilities are not clear. On one hand it is proposed that the metallic tip produces a local enhancement of the optical radiation similar to the well-known surface-enhanced Raman effect [2-4, 6]. On the other hand the possibility of thermal expansion and therefore of mechanical contact is also discussed [5].

In order to clarify this situation we have performed STM experiments in combination with the use of nanosecond laser pulses. Although a direct access to nanosecond time-resolved measurements is not possible with our present setup, we used different schemes of indirect measurements to learn about the interaction of the nanosecond laser pulse with the tip of a scanning tunneling microscope. From these experiments we conclude that thermal expansion of the tip cannot be neglected in experiments with nanosecond laser pulses but may even be the dominating mechanism involved.

\section{Experimental setup}

All experiments were performed on thin gold films $(150 \mathrm{~nm})$ on mica under ambient conditions with a home-built STM using etched PtIr tips. The response time of the current amplifier system is of the order of $50 \mu \mathrm{s}$. The amplifier showed a linear response in the measured current range of up to $10 \mathrm{nA}$. The setup allows the feedback mechanism to be switched off for a certain time. During this time an additional external voltage can be applied to the $z$ piezo in order to retract the tip in a defined manner. After the tip has been retracted the laser pulse illuminates the tip-surface gap. The time-resolved response of the tunneling current is recorded. In order to improve the signal-to-noise ratio the curves shown in the experimental part are typically averaged over 64 events.

For the illumination a mildly focused Q-switched Nd:YAG laser pulse $(0.1 \mathrm{~mm}$ diameter $)$ was used. The angle of incidence was $75^{\circ}$ with respect to the tip axis (Fig. 1). Both the fundamental at a wavelength of $1064 \mathrm{~nm}$ as well as the frequency-doubled pulse $(532 \mathrm{~nm})$ were applied with qualitatively identical results. Therefore, in the following, only results obtained with $\lambda=532 \mathrm{~nm}$ will be shown. The pulse width was $7 \mathrm{~ns}$ (FWHM). Volume absorbers allowed us to choose the proper energy density range for different experiments, whereas the fine tuning of the energy density was performed with the help of thin-film polarizers. The laser pulse intensity was controlled with an energy meter before attenuation. The pulse-to-pulse energy variation at the tip

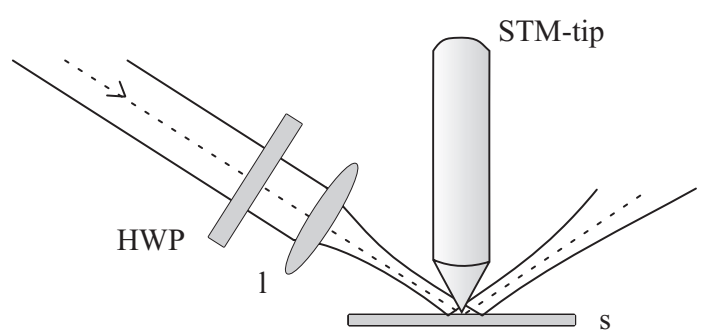

Fig. 1. Schematic diagram of the experimental setup. HWP, half-wave plate; 1 , lens; s, substrate 
position was around $5 \%$. For this measurement a $10-\mu \mathrm{m}$ pinhole was mounted in front of a PIN photodiode (FND 100, rise time $1 \mathrm{~ns}$ ) and the whole placed at the later position of the STM tip. The polarization of the incident laser light with respect to the tip could be adjusted with the help of a half-wave plate.

\section{Results}

In order to verify the possibility of nanostructuring and to define the interesting energy density range we increased the pulse energy until a nanostructure appeared on the surface after a pulse. Figure 2 shows the topography of a gold surface after (a) illumination with six, and (b) after the illumination with seven laser pulses of energy density $400 \mathrm{~mJ} / \mathrm{cm}^{2}$. In addition to the six hillocks already apparent in (a) a further hillock with a diameter of around $20 \mathrm{~nm}$ and a height of $5 \mathrm{~nm}$ appeared on the left side. This observation is similar to earlier studies [2-4] and will not be discussed further. We focus on dynamic measurements in the following. In order to be sure that the tip is not modified during the experiment all measurements were performed at densities at least one order of magnitude smaller than the threshold for nanostructure formation.

In the following experiments the retraction technique mentioned above was used to gain more insight into the ongoing processes. Figure 3 shows the principle of the measurement. At $t=-1.5 \mathrm{~ms}$ the feedback loop is switched off
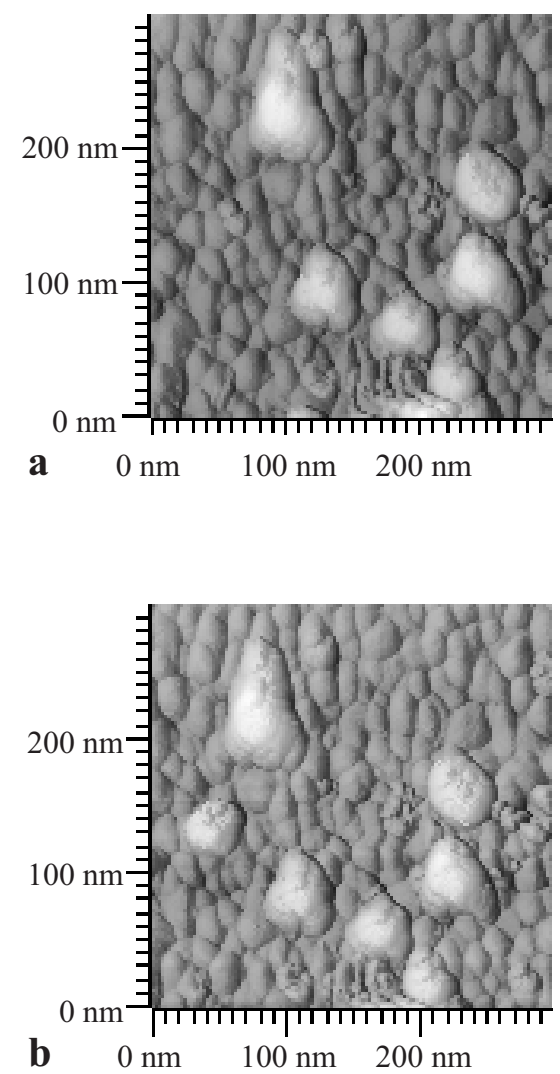

Fig. 2a,b. Surface topography $(300 \mathrm{~nm} \times 300 \mathrm{~nm})$ of an Au film a after the application of six and $\mathbf{b}$ after application of seven laser pulses of energy density $400 \mathrm{~mJ} / \mathrm{cm}^{2}$. In the upper left corner a new nanostructure appeared
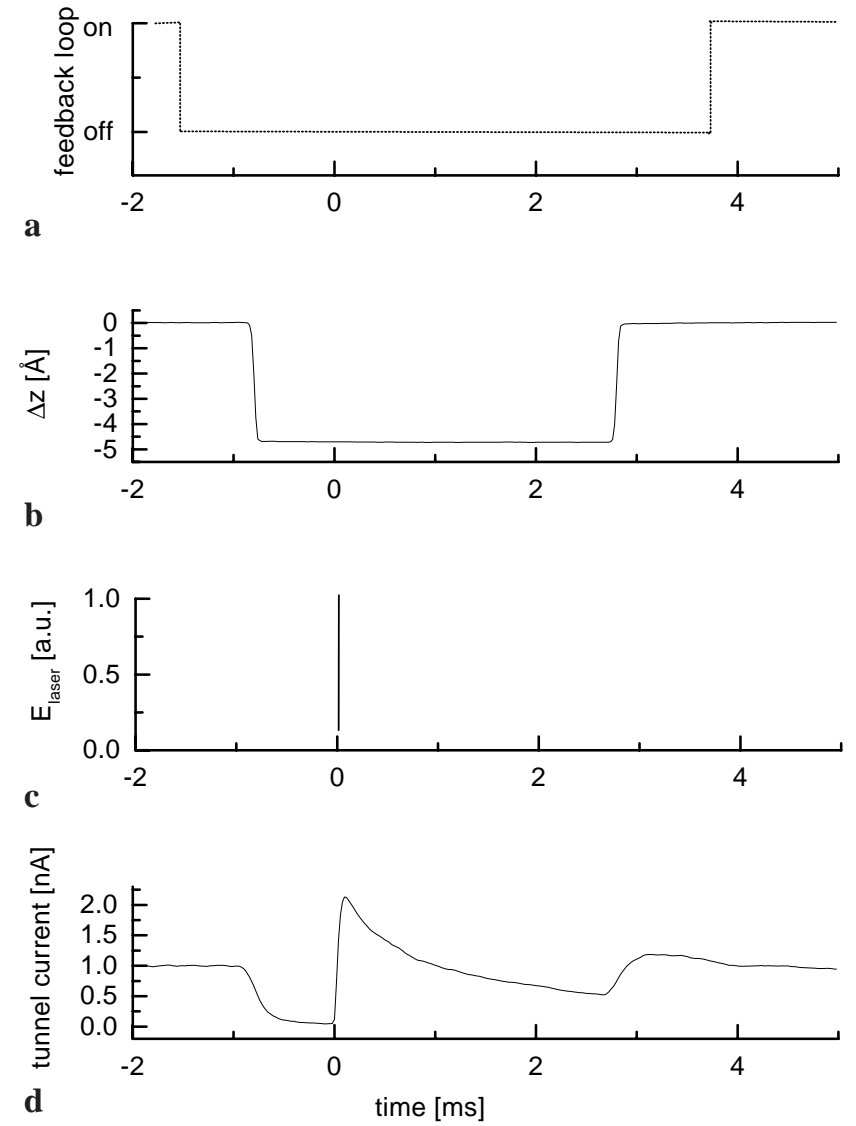

Fig. 3a-d. Principle of the timing of a retraction measurement at an energy density of $20 \mathrm{~mJ} / \mathrm{cm}^{2}$. a Feedback loop, b retraction amplitude, $\mathbf{c}$ laser pulse, and $\mathbf{d}$ tunneling current

(Fig. 3a). Then, by applying voltage to the piezo, the tip is retracted a certain distance, in this case $5 \AA$ [7] and fixed there for the next $3.5 \mathrm{~ms}$ (Fig. 3b). At $t=0$ the laser pulse (FWHM $7 \mathrm{~ns}$, pulse intensity $20 \mathrm{~mJ} / \mathrm{cm}^{2}$ ) hits the surface (Fig. 3c). Figure $3 \mathrm{~d}$ depicts the resulting tunneling current. During the retraction the tunneling current drops from the chosen set current of $1 \mathrm{nA}$ to zero. Upon illumination of the tunneling gap at $t=0$, the tunneling current shows a steep increase towards a maximum of $2.2 \mathrm{nA}$ and then a slow decrease back to zero on a ms timescale. After reapproaching the tip the current is first slightly higher than the set current and relaxes then towards the set current upon reactivation of the feedback loop.

We then performed the experiment for different retraction amplitudes at the same energy density. Some examples are shown in Fig. 4a,b. A clear decrease in the current peak is observed as the retraction amplitude is increased. Figure 4c shows the data of Fig. $4 \mathrm{~b}$ on a logarithmic scale. There, an almost linear behavior can be recognized.

The measurements were repeated for different laser energies. Upon plotting the extracted maximum current as function of retraction amplitude (Fig. 5a) it can be seen that the peak current increases with laser pulse intensity. On a logarithmic scale the same data reveal a linear behavior for all pulse energy densities (Fig. 5b). Different pulse energies result in a parallel shift of the curves. An additional energy input of $30 \mathrm{~mJ} / \mathrm{cm}^{2}$ results in an additional retraction amplitude of $0.6 \AA$. 

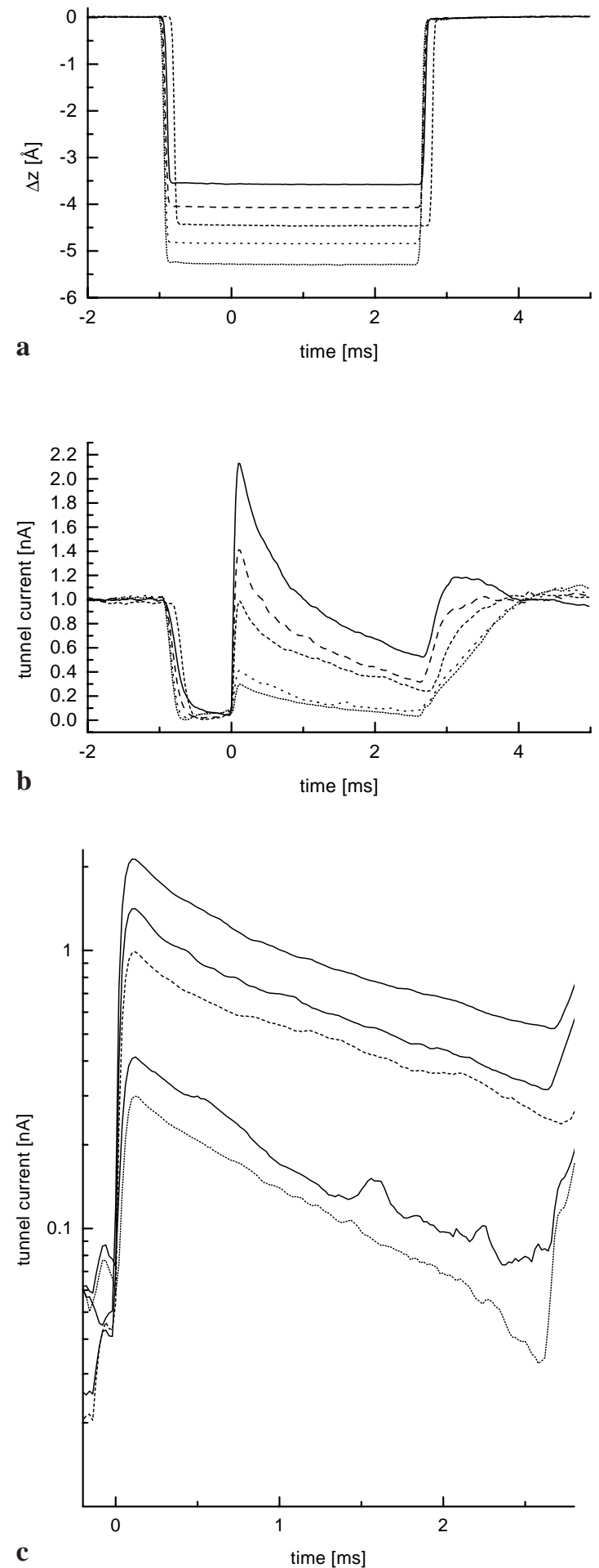

Fig. 4a-c. Transient tunneling current as a function of the retraction amplitude at an energy density of $20 \mathrm{~mJ} / \mathrm{cm}^{2}(\mathbf{a}, \mathbf{b})$. $\mathbf{c}$ displays the data of $\mathbf{b}$ on a logarithmic scale with a linear fit

Figure 6 shows 90 traces of the time-resolved tunneling current, where we studied the polarization dependence of the effect. The angle of the half-wave plate was tuned for $360^{\circ}$ in steps of $4^{\circ}$ starting parallel to the tip. Thus the polarization is changed for $720^{\circ}$. A strong polarization dependence of the effect is observed, similar to recent experiments [6]. This de-
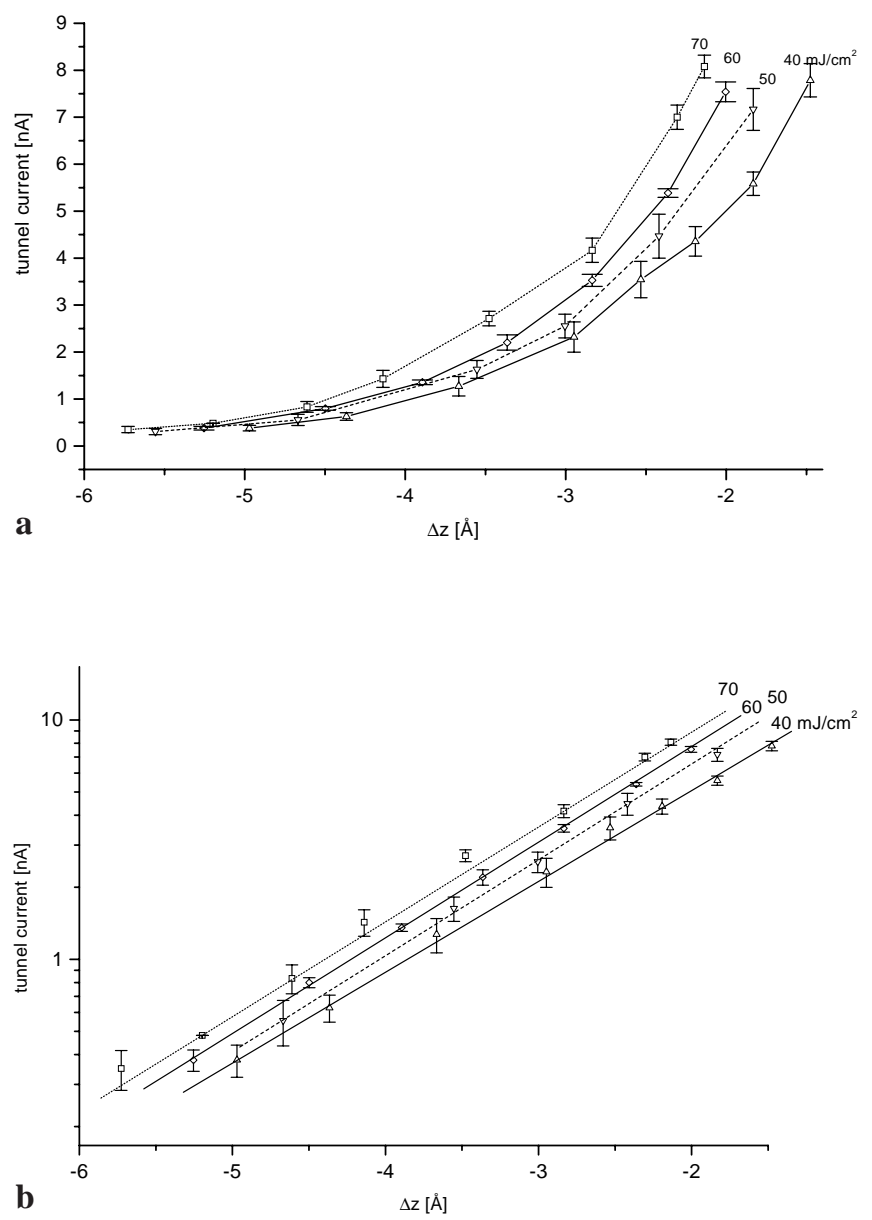

Fig. 5a,b. Maximum current $I_{\max }$ as a function of the retraction amplitude for different laser intensities. In $\mathbf{b}$ the data of $\mathbf{a}$ are displayed on a logarithmic scale

pendence appeared only as the laser focus hit the very end of the STM tip. Upon illuminating the tip some mm above the tunneling gap, the resulting tunneling current was considerably smaller and the polarization dependence disappeared. In the figure a thermal drift can also be observed, the absolute amplitude in the maximum is slightly increasing with time. This is explained by the fact that it took about $3 \mathrm{~h}$ to get these data as each single trace is averaged over 64 events.

All the measurements shown here were repeated with other tips of different materials. Whereas details of the described features may change, the overall features remain unchanged. The timescale of the decrease in the tunneling current changed between $2 \mathrm{~ms}$ and $0.5 \mathrm{~ms}$, and the retraction amplitude between 0.02 and $0.5 \AA /\left(\mathrm{mJ} / \mathrm{cm}^{2}\right)$. The measured examples showed a correlation with the opening angle of the tip. Nevertheless this was not studied systematically.

\section{Discussion}

In the following we will show that all experimental results fit in the scheme of thermal expansion of the tip and the sample. The first striking result is the timescale observed in the experiment. Whereas the current increase (Fig. 3) cannot be analyzed because of the limited response time of the electronics, the current decrease occurs on a timescale of ms, which 


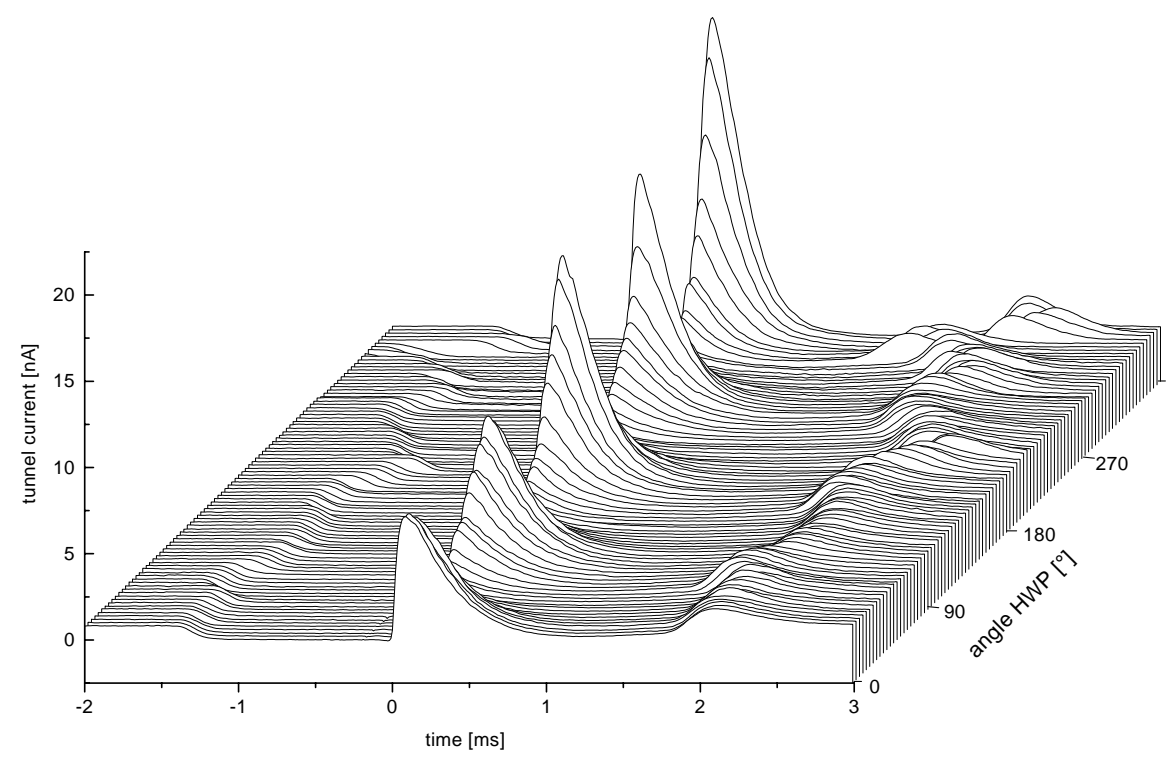

Fig. 6. Retraction experiment as a function of the polarization of the laser pulse for an energy density of $20 \mathrm{~mJ} / \mathrm{cm}^{2}$ is five orders of magnitude slower than the laser pulse duration $(7 \mathrm{~ns})$. In particular the ms timescale is well above the response time of the current amplifier system $(50 \mu \mathrm{s})$. Therefore a field-induced tunneling current can be excluded for the explanation of the time response of the tunneling current.

In principle the data of Fig. 4a,b allow a direct estimation of the thermal expansion of the tip and sample, which can be estimated to be some $\AA$ for the energy density used. However, a more detailed analysis is possible. For that purpose, first the consequences of the limited time-resolution must be considered. From Fig. 4c we can deduce that the tip retracts almost linearly upon cooling, as the current on a logarithmic scale is proportional to $z$. This makes it possible to extrapolate from $I_{\max }$, which is measured at $t=150 \mu \mathrm{s}$, to the end of the laser pulse at $t=10 \mathrm{~ns}$, where the maximum expansion is expected [5]. Concerning the data it is resonable to assume that the deviation from the maximum expansion at $t=150 \mu \mathrm{s}$ is below $10 \%$. Thus $I_{\max }$ can be analyzed further on.

The data of Fig. 5 include additional information. The linear dependence of $\Delta z$ on the $\log I_{\max }$ for each energy density reflects the fact that this measurement is in principle nothing other than a modified $I(z)$ measurement. At the moment when $I_{\max }$ is measured $(t=150 \mu \mathrm{s})$ the distance to the surface is the retraction amplitude $\Delta z$ minus the expansion of tip and surface. Thus $I_{\max }(\Delta z)$ reflects the exponential behaviour of the tunneling current. Since this is true for each energy density it is possible to extract the absolute values of expansion from these measurements even though the absolute tip surface distance is not known exactly. From the parallel shift of the different energy densities we get a value of $0.02 \AA / \mathrm{mJ} / \mathrm{cm}^{2}$ for the tip/sample combination used here. Furthermore the lines are roughly equidistant, which allows us to conclude that the absorption and expansion are linear in this energy density regime. This finding agrees with theoretical considerations by Park et al. [9]. Consequently the expansion can now be deduced for each energy density.

With this value we can try to get an estimation of the temperature increase during the pulse for the maximum energy density $\left(70 \mathrm{~mJ} / \mathrm{cm}^{2}\right)$ used in this experiment. For a first approximation it can be assumed that the thermal expansion of the substrate is small compared to that of the tip [5]. The heat diffusion length in platinum is of the order of $l_{\text {diff }}=0.7 \mu \mathrm{m}$ on the timescale of the laser pulse. As already discussed by Ukraintsev et al. [5] not the whole illuminated area does contribute to the thermal expansion of the tip. For a tip with an opening angle of $\alpha=30^{\circ}$ we get an effective contribution length in the order of $d_{\text {eff }}=\left(l_{\text {diff }} / 2\right) /(\tan \alpha / 2)=1.3 \mu \mathrm{m}$. Taking an expansion coefficient of $8.9 \times 10^{-6}$ for platinum the expansion of $1.4 \AA$ results in an average temperature increase of $12 \mathrm{~K}$ for this tip, which is surprisingly small. For other tips we got higher values of expansion. There the resulting temperatures would be a factor of 25 higher.

For such a small temperature increase neither a detectable change of the tunneling possibility (due to a change of the Fermi function) nor a detectable thermovoltage should be expected. This is confirmed by the symmetrical dependence $\left|I_{\max }\right|$ on the applied tunneling voltage for $|U|<0.3 \mathrm{~V}$ (not shown).

The polarization dependence of $I_{\max }$ can be explained by the polarization dependence of the absorbed energy. The very end of the tip can be assumed to be an ellipsoid and then the absorption depends on polarization $[9,10]$. Consequently the polarization dependence should disappear almost completely as the shaft of the tip is illuminated, as observed in the experiment.

\section{Conclusion}

Our experiments have shown that even at energy densities one order of magnitude below the threshold for the appearance of nanostructures, the thermal expansion of the tip cannot be neglected. The tip is observed to expand linearly with the illumination energy density and retracts on a timescale of $\mathrm{ms}$ [12]. For the energy densities used in the nanostructuring experiments here as well as in the literature we estimate thermal expansion in the order of nanometers. This could imply that mechanical contact between tip and surface is established upon the illumination. Indeed our first measurements of the gap voltage indicate quantized contact resistance [11]. There- 
fore we have to question if the field enhancement at the tip is the dominating effect for the occurrence of nanostructures upon illumination with nanosecond pulses. Hence we started to use fs pulses where the scenario could be expected to change.

\section{References}

1. P. Avouris (Ed.): Atomic and Nanometer-Scale Modification of Materials: Fundamentals and Applications, NATO ASI Series e, Vol. 239 (Kluwer, Dordrecht 1993)

2. A.A. Gorbunov, W. Pompe: Phys. Status Solidi A 145, 333 (1994)

3. K. Dickmann, J. Jersch: Laser Optoelektronik 27, 76 (1995)
4. J. Jersch, K. Dickmann: Appl. Phys. Lett. 68, 868 (1996)

5. V.A. Ukraintsev, J.T. Yates Jr.: J. Appl. Phys. 80, 2561 (1996)

6. J. Jersch, F. Demming, K. Dickmann: Appl. Phys. A 64, 29 (1997)

7. The $z$-piezo was calibrated using monatomic steps on the Au surface

8. S.H. Park, N.M. Miskovsky, P.H. Cutler, E. Kazes, T.E. Sullivan: Surf. Sci. 266, 265 (1992)

9. H.C. van de Hulst: Light Scattering by Small Particles (Dover, New York 1957)

10. C.F. Bohren, D.R. Huffmann: Absorption and scattering of light by small particles (Wiley, New York 1983)

11. J. Boneberg, M. Tresp, M. Ochmann, H.-J. Münzer, P. Leiderer: to be published in Appl. Phys. A

12. Note added in proof: The same conclusions were recently given by I. Lyubinetsky, Z. Dohnálek, V.A. Ukraintsev, J.T. Yates, Jr.: J. Appl. Phys. 82, 4115 (1997) 